Brickner, M.S. and Foyle, D.C. (1990). Proceedings of the Human Factors Society 34th Annual Meeting, 1567-1571. Santa Monica, CA: HFES.

\title{
FIELD OF VIEW EFFECTS ON A SIMULATED FLIGHT TASK WITH HEAD-DOWN AND HEAD-UP SENSOR IMAGERY DISPLAYS
}

\author{
Michael S. Brickner \\ PAMAM Human Factors Engineering Ltd. \\ Ramat-Hasharon, Israel \\ David C. Foyle \\ NASA Ames Research Center \\ Moffett Field, CA
}

\begin{abstract}
When pilots fly an aircraft with sensor imagery rather than direct vision, their instantaneous field of view (FOV) is restricted severely. This limitation has been identified as a major human factors problem in aviation and has fostered efforts to develop displays with much wider FOVs than are currently available. Two experiments are reported investigating FOV effects in simulated flights with sensor imagery appearing on a Head-Down Display (HDD) and a Head-Up Display (HUD). The outside world was viewed via a simulated sensor image with a 25,40 , or 55 deg FOV, while the surrounding world was dark and provided no additional information. The subjects task was to fly a slalom course without hitting the pylons or missing the intervals between pylons. The results indicate significant effects of FOV on both hits and misses for both the HDD and the HUD. In addition, performance was significantly affected by the speed of flying and the level of training. With the narrow FOV, subjects flew closer to the pylons than with the wide FOV and hit the pylons more often. These results are interpreted as an indication that subjects perceived the sensor display as the entire world rather than as a window into the world. This effect was somewhat smaller with the HUD than with the HDD, possibly because the HUD better represented a window into the world. The differences between the HUD and the HDD, however, did not reach statistical significance.
\end{abstract}

\section{INTRODUCTION}

In most civil and military flight operations, pilots rely on visual cues to maintain situational awareness. The normal binocular field of view (FOV) of the human observer is approximately $+/-100$ deg horizontal and $+/-60$ deg vertical (Harrington, 1964). The field of regard, which takes into consideration eye and head movements, covers most of the sphere around the observer, however, the actual FOV, from the pilot s eye position in the cockpit, is significantly limited by the structure of the aircraft and by personal gear (e.g., helmet, night-vision goggles). Providing wider FOVs is one of the primary design goals of advanced military aircraft. For example, the bubble canopy of the F-16 fighter aircraft and the large canopy of the Apache AH-64 attack helicopter are designed to offer the pilot larger unrestricted FOVs than those provided by previous generations of aircraft.

Some flight conditions, however, impose constraints that significantly restrict the pilots FOVs. Light intensifiers and thermal imaging devices, allow pilots to fly during the night and other low visibility conditions, presenting the pilot a window of relatively clear visibility into an otherwise low-visibility world. The sensor image may be presented on a panel-mounted head-down display (HDD), a head-up display (HUD) or a head (helmet) mounted display. The visual field provided by HDDs is typically $8-15 \mathrm{deg}$ (horizontal); conventional HUDs extend 12-18 deg; advanced holographic HUDs provide up to 30 deg (Wurfel, 1984). Helmet-mounted displays and night-vision goggles have immediate horizontal FOVs of up to $40 \mathrm{deg}$ (Hart \& Brickner, 1989). The restricted immediate FOV has been identified as a major human factors problem in aviation, even when head movements are unlimited (e.g., with nightvision goggles; Brickner, 1989a). Recently, in order to solve these problems, extensive efforts have been made to develop displays for both simulators and real aircraft with much wider FOVs than are currently available (Shenker, 1987).

Little research, however, has been conducted to examine directly the effects of reduced FOV on flight performance or to establish FOV criteria for various types of tasks. Most FOV research has focused on simulator visual systems (in which the size of the FOV is related to simulation fidelity rather than to pilot performance; Welch \& Kruk, 1986). Other research has focused on problems related to specific display systems (e.g., large FOV artificial horizons; Malcolm, 1984), their role in movement detection (Wright, 1987) and in inducing vection (Huang \& Young, 1988), and the specific functions of focal (foveal) versus ambient (peripheral) vision (Leibowitz, Shupert \& Post, 1984).

The two experiments reported herein simulated a helicopter flight situation in which the outside world is seen through a sensor image with a limited FOV while the surrounding world is dark and provides no additional information. The simulated sensor image display was presented on either a HDD or a HUD. Three different FOVs were provided for each of the displays.

\section{EXPERIMENT I: HDD}

Method

Subjects. Eight male subjects served as paid participants in the experiment. All reported 20/20 corrected or uncorrected vision.

Apparatus and Display. A spring-loaded joystick was used to control a helicopter flight through a world view generated by a Silicon Graphics 3130 presented on a 19 display (Fig. 1,2). The center of the screen was 
approximately $15 \mathrm{deg}$ below the eye height of the subject. The scene was monochrome and simulated some of the features of thermal images. It consisted of a take-off site, eight pylons in a row, entrance and exit windows, and, in some conditions, a superimposed grid on the ground. The distance between pylons varied between 400-800 ft with an average of $600 \mathrm{ft}$. Three different slalom courses were used in a random order to prevent the subjects from developing a manual control motor scheme.

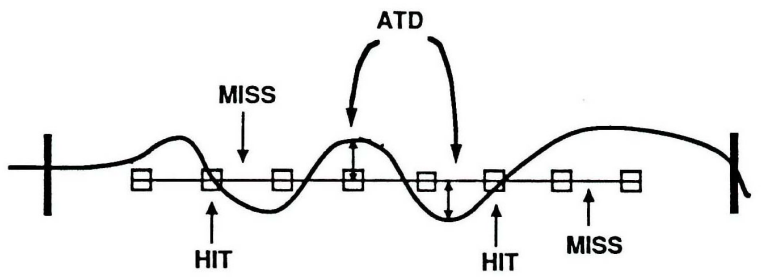

Figure 1. Plan view of slalom course (not to scale.)

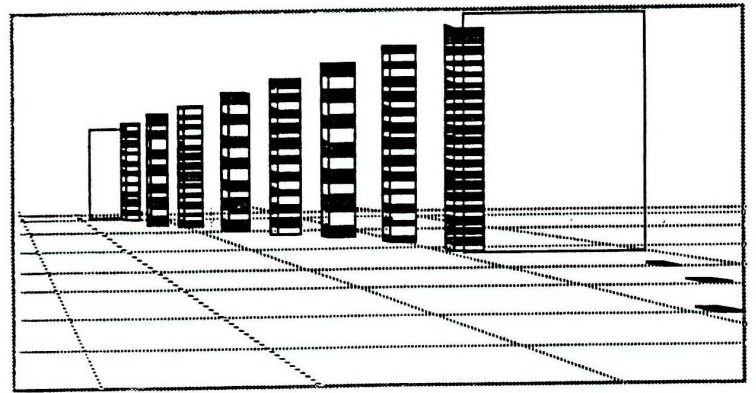

Figure 2. Stylized perspective view of slalom course.

Procedure. The subjects were asked to ascend from the landing site, climb to an altitude of 100 feet, fly through the entrance window and then complete the slalom course. The trial ended when the helicopter was flown through the exit window (Fig. 1, 2). The subjects were asked to avoid hitting the pylons and missing any of the intervals between them. They were also advised to fly the most efficient course by going as close to the pylon as they felt safe, thus shortening the time required to complete each slalom course. It was emphasized, however, that avoiding hits and misses was much more important than saving time. A beep sounded whenever a pylon was hit, but the helicopter did not crash. Full feedback on the number of hits, misses and flight duration was given to the subject at the end of each trial. During data analysis, the actual flying distance from the pylons (average turn distance (ATD); Fig. 1) was used as a measure of flight efficiency, instead of flight duration.

Independent Variables. Three independent variables were manipulated:

a. FOV. Three FOVs were used: 25,40 , and $55 \mathrm{deg}$ horizontal (the vertical dimensions were 19, 30 and $41 \mathrm{deg}$, respectively).

b. Speed. Two flying speeds were tested: 82 and 109 knots.

c. Ground texture. A grid superimposed on the ground was either present or absent.
Dependent variables. Three measures were used (see Fig. 1 for examples).

a. Hits. Number of hits of pylons.

b. Misses. Number of misses of intervals between pylons.

c. ATD. Average turn distance (ATD) around the pylons.

ATD is the average maximal distance between the helicopter and the row of pylons at each turning point.

For the different FOVs, the subjects viewing distance of the display was varied such that a 1:1 magnification of the world representation was maintained. The distances between the subjects eyes and the center of the screen were 35,50 , and $80 \mathrm{~cm}$ for the 55, 40, and $25 \mathrm{deg}$ FOV, respectively.

Experimental Design. A within-subject design was used. Each subject performed a total of 72 trials consisting of six replications of the 12 experimental conditions created by the three independent variables $(3 \times 2 \times 2)$. The order of conditions within each block of 12 trials was counterbalanced.

$\underline{\text { Results }}$

A four-way ANOVA (FOV x speed $\mathrm{x}$ grid $\mathrm{x}$ replication) was performed for each of the dependent variables.

Number of hits. There were significant effects of FOV $(\mathrm{F}(2,14)=80.88, \mathrm{p}<0.001)$; grid $(\mathrm{F}(1,7)=6.32, \mathrm{p}<0.05)$; and an interaction between FOV and speed $(\mathrm{F}(2,14)=5.41$, $\mathrm{p}<0.02$ ).

Number of misses. There were significant effects of FOV $(\mathrm{F}(2,14)=6.91, \mathrm{p}<0.01)$; speed $(\mathrm{F}(1,7)=21.53, \mathrm{p}<0.01)$; and replications $(\mathrm{F}(5,35)=6.72, \mathrm{p}<0.001)$.

ATD. There were significant effects of FOV $(\mathrm{F}(2,14)=22.02, \mathrm{p}<.001$; speed $(\mathrm{F}(1,7)=27.91, \mathrm{p}<.01)$; and replications $(\mathrm{F}(5,35)=4.20, \mathrm{p}<.01)$.

\section{$\underline{\text { Discussion }}$}

The results reveal some differential effects of the independent variables on the measures of performance and reflect some of the performance strategies used by the subjects. Both speed and training (i.e., replications) had a larger effect on misses than on hits (Fig. 3); subjects tended to avoid hits, rather than avoid misses despite instructions that both were equally important. These results are consistent with previous findings (Brickner, 1989b). However, the subjects were not able to maintain a constant level of performance with reduced FOV (Fig. 5).

The effect of FOV on ATD (Fig. 7) indicates that subjects did not completely adapt their flying strategy to the changing FOV. Although they knew that the display represented only a window into the world, they tended to act as if the window was the whole world. Thus, the smaller the FOV, the closer they flew to the pylons, resulting in three times the number of hits for $25 \mathrm{deg}$ than for $55 \mathrm{deg}$ FOV; see Fig. 5). 


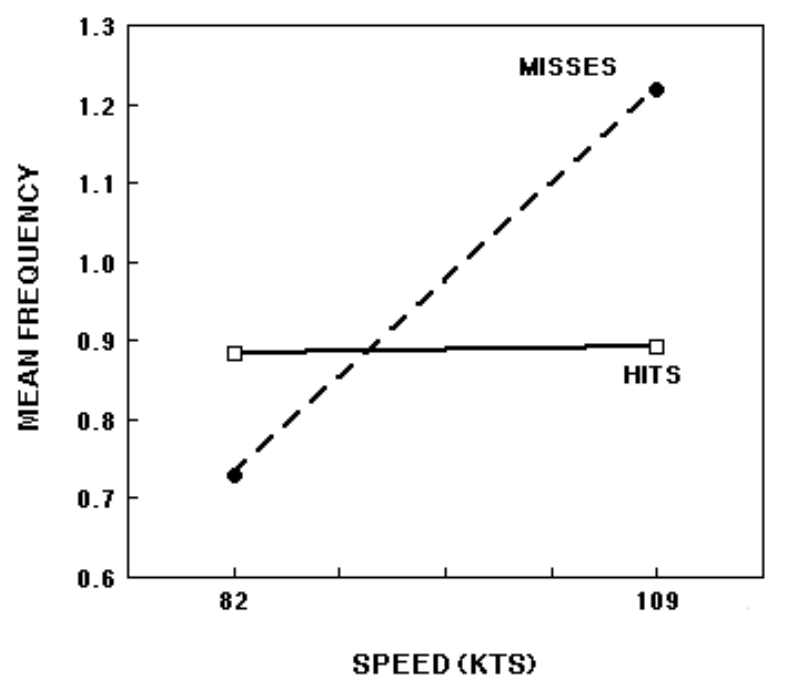

Figure 3. Effect of flight speed on hits and misses.

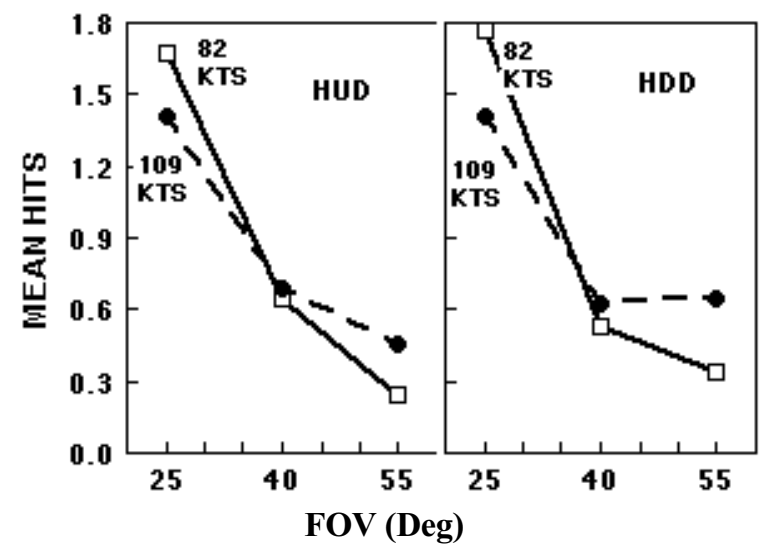

Figure 4. Effect of FOV and speed on mean hits for the HUD and HDD.

The presence of a grid superimposed on the ground significantly reduced the number of hits, indicating that added texture information improved spatial orientation. It was expected that the grid would be more important with a narrow FOV because spatial orientation would be more difficult. However, the grid and FOV effects did not interact significantly for either hits or misses, and the grid had no effect on misses.

The strong effects of FOV on ATD may be attributed to the nature of the HDD. The HDD was surrounded by the frame of the monitor and the instrument panel and may not have been perceived as a window into the world. Similarly, it has been shown that HDD displays create stronger under-estimations of size and distance than HUD type displays (Roscoe, 1985). It was, therefore, hypothesized that FOV effects on ATD should be less with a HUD because of the direct representation of the surrounding outside world in the display. The second experiment was designed to test this hypothesis.

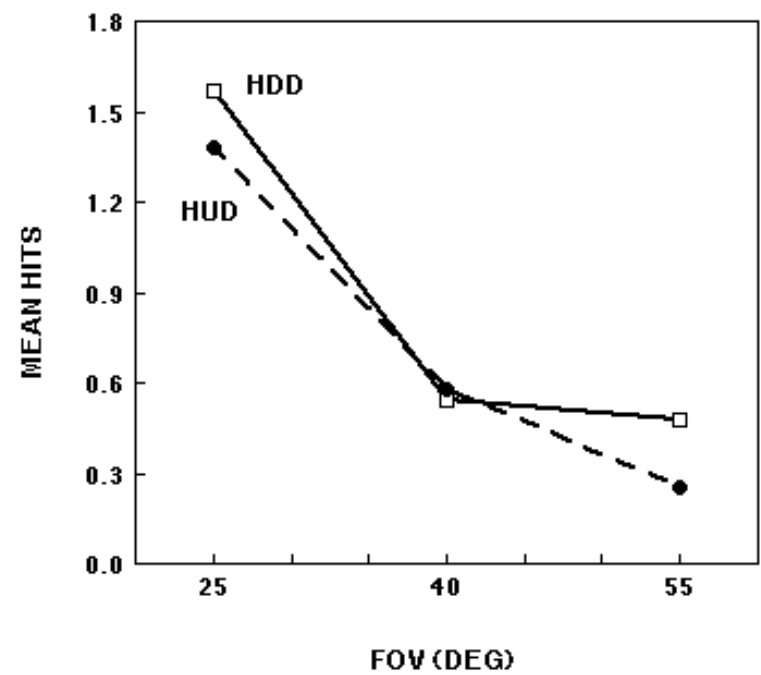

Figure 5. Effect of FOV on hits for the HUD and HDD.

EXPERIMENT II: HUD

The second experiment used the same flight task as the first. However, the world view was presented on a wide screen display which simulated a Head-Up Display (HUD) in the sense that the presence of the dark outside world, surrounding the bright sensor image was clearly represented on the unused peripheral parts of the wide screen. The center of the screen was approximately at eye height.

Method

Experiment II was identical to Experiment I except for the following: The display was projected on an Electrohome ECP 3000 wide-screen display with the subjects eyes 145 $\mathrm{cm}$ from the center of the screen. FOV was altered by changing the size of the graphic viewport on the screen (in software) and were identical to Experiment I (25, 40 and 55 deg horizontal). The total area of the wide screen extended approximately $80 \times 65(\mathrm{H} \times \mathrm{V}) \mathrm{deg}$. The joystick used in Experiment II was not as strongly centered as the one used in the first experiment. As a result, the helicopter was slightly more difficult to control. Eight additional male subjects served as paid participants. All reported 20/20 corrected or uncorrected vision.

$\underline{\text { Results }}$

As in Experiment I, a four-way ANOVA was performed for each of the dependent variables.

Number of hits. FOV $(\mathrm{F}(2,14)=93.97, \mathrm{p}<0.001$; replications $(\mathrm{F}(5,35)=5.28, \mathrm{p}<0.001)$; and the interaction of FOV and speed $(\mathrm{F}(2,14)=5.34, \mathrm{p}<0.02)$ were statistically significant.

Number of misses. FOV $(\mathrm{F}(2,14)=8.86, \mathrm{p}<0.01)$; speed $(\mathrm{F}(1,7)=71.46, \mathrm{p}<0.001)$; and replications $(\mathrm{F}(5,35)=10.41$, $\mathrm{P}<0.001)$ were significant.

ATD. FOV $(\mathrm{F}(2,14)=45.10, \mathrm{p}<.001)$; speed $(\mathrm{F}(1,7)=26.26, \mathrm{p}<.01)$; replications $(\mathrm{F}(5,35)=7.44, \mathrm{p}<.001)$; and the FOV, ground texture, replication interaction $(\mathrm{F}(10,70)=2.08, \mathrm{p}<.05)$ had significant effects 
$\underline{\text { Discussion }}$

The results of Experiment II were quite similar to those of Experiment I. As in the first experiment, the FOV had a significant effect on hits. The grid effect, however, did not replicate. On the other hand, there was a significant replication (training) effect in the second experiment only. The reason for the significant training effect may be related to the more difficult helicopter control in Experiment II. Whereas the overall average hit rate was higher with the HDD (0.89) than with the HUD (0.85), the average of the first two replications was considerably better with the HDD (0.94) than with the HUD (1.08).

The interaction between FOV and speed was significant in both experiments with very similar patterns of results (Fig. 4): For the wide FOV, the hit rate was lower at low speed, whereas with the narrow FOV, hit rates were lower at high speed. Thus, the total effect of FOV on hit rate was generally larger at the slow flying speed than at the high speed. This consistent and significant effect seems to be counter-intuitive; one might expect high speed and narrow FOV to have an accumulating rather than a contradicting effect on hits. It is possible that with the narrow FOV, in which visual cues were sometimes lacking, subjects tried to respond by developing motor schemes which were more effective at high speed (although the experiment tried to prevent the development of such schemes by altering the distances between the pylons).

In both experiments, the same variables had significant effects on misses and on ATD. It was hypothesized that the FOV effect on ATD would be smaller with the HUD than it was with the HDD. Although the results point in this direction, FOV still had a significant ATD effect on performance with the HUD.

\section{COMPARISON OF EXPERIMENTS I AND II}

In order to compare performance with the HDD and the HUD, the results of both experiments were analyzed together using a five-way mixed ANOVA (FOV, speed, display (HDD or HUD), replications, grid). In order to minimize the effect of replication (training), the common analysis was performed on the last four (out of six) experimental replications only.

\section{$\underline{\text { Results }}$}

Number of hits. FOV $(2,28)=64.63, \mathrm{p}<0.001$, replications $(\mathrm{F}(3,42)=6.20, \mathrm{p}<0.01)$, grid $(\mathrm{F}(1,14)=11.65$, $\mathrm{p}<0.01)$, the interaction of replications with FOV $(\mathrm{F}(6,84)=3.32, \mathrm{p}<0.01)$, and of FOV with speed $(\mathrm{F}(2,28)=11.09, \mathrm{p}<0.001)$ were significant. The display effect did not reach statistical significance $(\mathrm{F}(1,14)=2.20$, $\mathrm{p}=0.16)$.

Number of misses. FOV $(\mathrm{F}(2,28)=12.38, \mathrm{p}<0.001)$, speed $(\mathrm{F}(1,14)=99.41, \mathrm{p}<0.001)$, and replications $(\mathrm{F}(3,42)=4.15, \mathrm{p}<0.02)$, were significant. The display effect did not reach statistical significance $(\mathrm{F}(1,14)=2.15, \mathrm{p}=0.16)$.

ATD. FOV $(\mathrm{F}(2,28)=45.23, \mathrm{p}<0.001)$, speed
$(\mathrm{F}(1,14)=53.61, \mathrm{p}<0.001)$ and replications $(\mathrm{F}(3,42)=3.99$, $\mathrm{p}<0.02$ ) had significant effects on ATD. Although the grand mean of the ATD was considerably larger for the HDD (167 $\mathrm{ft})$ than for the HUD $(144 \mathrm{ft})$, this difference did not reach statistical significance $(F(1,14)=2.52, p=0.13)$.

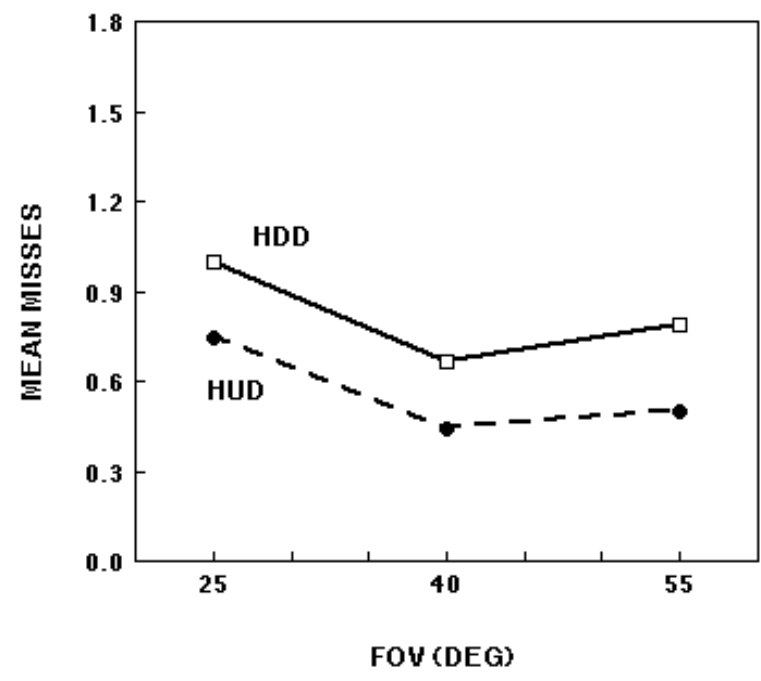

Figure 6. Effect of FOV on hits for the HUD and HDD.

\section{GENERAL DISCUSSION}

In general, the combined results of the two experiments confirm and strengthen the individual result of each experiment. The comparison of the two displays showed that overall performance with the HUD was superior to performance with the HDD; there were fewer hits (Fig. 5), fewer misses (Fig. 6), and a smaller ATD (Fig. 7) with the HUD. In view of the fact that each of the three variables was close to statistical significance, a larger sample size may have yielded significant display differences. Also, the increased control difficulty in Experiment II (the HUD conditions) probably attenuated display-specific differences. The superiority of the HUD is in accord with other studies which compared head-up and head-down performance on other types of flight maneuvers, such as landing (Haines, Fischer \& Price, 1980).

The presence of ground texture helped to reduce the number of hits significantly. This result is in accord with previous findings which demonstrated the usefulness of gridtype ground textures in maintaining spatial position (Johnson, Bennett, O Donnell \& Phatak, 1988). It is not clear, however, why the grid did not significantly improve misses and ATD. The texture provided the necessary visual cues for estimating the location of the pylons when they were out of sight and could, in principle, help to reduce the rate of misses. The grid also provided a uniform size scale which could be used to maintain a relatively constant ATD. Clearly, this source of visual information was not exploited to its fullest extent. Perhaps more practice was needed for the subjects to make more efficient use of the texture. 


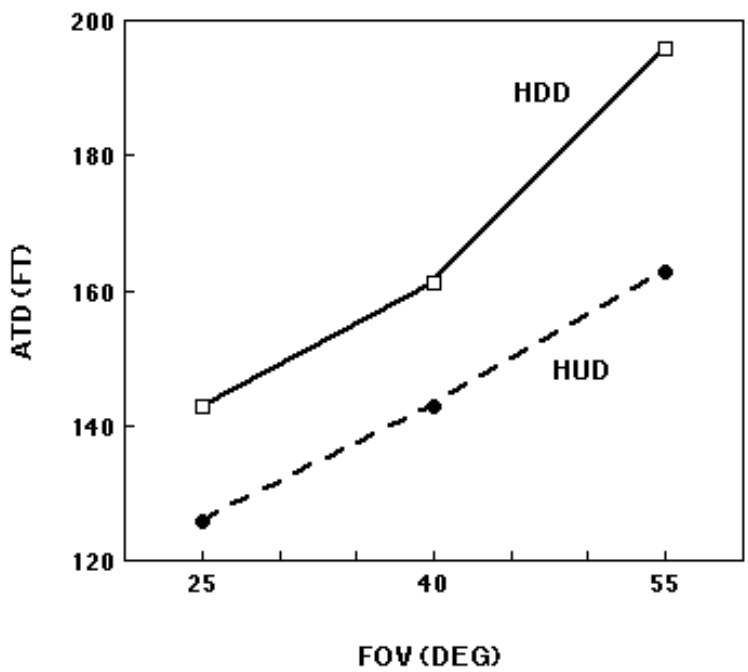

Figure 7. Effect of FOV on ATD for the HUD and HDD.

The effect of FOV on ATD (Fig. 7) reconfirmed the conclusion that subjects did not completely adapt their flying strategy to the changing FOV and tended to act as if the display represented the whole world rather than a bright window into the dark world. Thus, the smaller the FOV the closer they flew to the pylons (Fig. 7). This resulted in 3-4 times more hits for the $25 \mathrm{deg}$ than for the $55 \mathrm{deg}$ FOV (Fig. 5). It was hypothesized that the effect of FOV on ATD would be smaller for the HUD because it directly represented the surrounding outside world. The results are in the predicted direction, but did not reach statistical significance. Also, type of display and FOV did not interact. Thus, the general finding holds for both HUD and HDD.

The present research clearly demonstrated some of the major limitations of limited FOV displays and the advantages of larger displays. In addition, the fact that subjects tended to limit their maneuvering to the visible world may have important implications for training pilots to fly aircraft with sensor image displays. Further research is recommended to test whether experienced pilots are more efficient at using sensor imagery and limited FOV displays and whether they may be able make better use of ground texture than the naive subjects used in the present research.

\section{ACKNOWLEDGMENTS}

The research was completed while the first author was a National Research Council Research Associate at NASA Ames Research Center.

\section{REFERENCES}

Brickner, M.S. (1989a). Helicopter flights with night vision goggles - Human factors aspects (NASA TM101039), Moffett Field, CA: NASA.

Brickner, M.S. (1989b). Apparent limitations of head-up displays and thermal imaging systems. In Proceedings of the Fifth International Symposium on Aviation Psychology, Vol. I, Columbus, OH: Ohio State U, 703-707.

Haines, R.F., Fischer, E. \& Price, T.A. (1980). Head-up transition behavior of pilots with and without head-up display in simulated low-visibility approaches, (NASA TP-1720), Moffett Field, CA: NASA.

Harrington, D.O. (1964). The visual field. St. Louis: Mosby.

Hart, S.G., and Brickner, M.S. (1989). Helmet-mounted pilot night vision systems: Human factors issues. In Spatial Displays and Spatial Instruments (NASA CP10032), Moffett Field, CA: NASA, (13)1-(13)21.

Huang, J.K. \& Young L.R. (1988). Visual field influence on manual roll and pitch stabilization. Aviation, Space, and Environmental Medicine, 59, 611-619.

Johnson, W.W., Bennett, T.C., O Donnell, K. \& Phatak, A.V. (1988). Optical variables useful in the active control of altitude. In Proceedings of the $23^{\text {rd }}$ Annual Conference on Manual Control, Cambridge MA: MIT.

Leibowitz, H.W., Shupert, C.L. \& Post, R.B. (1984). The two modes of visual processing: Implications for spatial orientation. In Peripheral Vision Horizon Display (PVHD), NASA CP-2306, Moffett Field, CA: NASA, 41-44.

Malcolm, R. (1984). The Malcolm horizon - history and future. In Peripheral Vision Horizon Display (PVHD), NASA CP-2306, Moffett Field, CA: NASA, 11-40.

Roscoe, S.N. (1985). Bigness is in the eye of the beholder. Human Factors, 27, 615-636.

Shenker, M. (1987). Optical criteria for binocular helmetmounted displays. SPIE Display System Optics, 778, 70-78.

Welch, B.L. (1986). Engineering and human visual consideration in development of a fiber optic helmet mounted display. In Advances in Flight Simulation - Visual and Motion Systems Proceedings, London, Royal Aeronautical Society, 295-331.

Wright, M.J. (1987). Spatiotemporal properties of grating motion detection in the center and the periphery of the visual field. Journal of the Optical Society of America, 4, 1627-1633.

Wurfel, H.G.F. (1984). F-16 and F-10 diffraction optics head-up display (HUD) flight test evaluation. In AGARD Flight Test Technology HC A16/MF A01, Brussels, Belgium: AGARD. 